\title{
PROPOSTA DE METODOLOGIA PARA PLANEJAMENTO DE MANUTENÇÃO INDUSTRIAL*
}

Rodrigo Costa dos Santos ${ }^{1}$ Adauto Martins de Assis ${ }^{2}$

\section{Resumo}

O objetivo deste artigo é apresentar uma proposta de metodologia para auxílio na fase de planejamento de um projeto de manutenção, objetivando a redução de falhas no mesmo. Tal estudo possui relevância, pois quando se trata de manutenção preventiva o custo é sempre considerável para qualquer empresa, uma vez que para realização as máquinas devem ser paradas e recursos humanos e materiais devem ser disponibilizados, desta forma o planejamento deve ser feito de maneira que sejam utilizados apenas os recursos necessários e suficientes para manter a qualidade dos equipamentos. A metodologia se embasa na teoria geral de projetos e utiliza de maneira sequenciada e estratégica ferramentas já aplicadas no mundo da manutenção para determinar as oito fases personalizadas para um evento de manutenção. A aplicação da metodologia facilita o trabalho do planejador o que consequentemente viabiliza a sua entrada no meio industrial, posto que a metodologia é altamente simples e sequenciada, o planejador não precisa conhecer com detalhes a teoria de projetos para utiliza-la. Portanto, com objetivo de facilitar e sequenciar o planejamento da manutenção afim de reduzir falhas, a metodologia trabalha de maneira forte a análise e gestão de riscos e definição de cronogramas em projetos.

Palavras-chave: Metodologia; Manutenção; Planejamento; Projeto.

\section{PROPOSAL FOR A METHODOLOGY FOR INDUSTRIAL MAINTENANCE PLANNING}

\section{Abstract}

The purpose of this article is to present a proposal for a methodology to support in the planning phase of a maintenance project, with focus on reduction of failures. This study have relevance because whenever it is a question of preventive maintenance, the cost is always considerable for any company since to carry out, the machines must be stopped and human and material resources must be made available, in this way the planning must be done in such a way that only the necessary and sufficient resources are used to maintain the quality of the equipments. The methodology is based on the general theory of project and uses tools already applied in the maintenance world in a sequenced and strategic way to determine the eight customized phases for a maintenance event. The application of the methodology makes the planner's work easier which consequently enables it to enter the industrial environment, given that the methodology is highly simple and sequenced the planner need to know in details the theory of projects to use it. Therefore, in order to facilitate and sequencing maintenance planning to reduce failures, the methodology works in a hard way the analysis and management of risks and definition of schedules in a maintenance project.

Keywords: Methodology; Maintenance; Planning; Project.

1 Engenheiro Mecânico, Mestrando em Engenharia Mecânica, aluno do programa de pós-graduação em Engenharia Mecânica da Universidade Federal Fluminense, Volta Redonda, Brasil

2 Doutor em Eng metalúrgica, Professor, Dep. Engenharia Mecânica da Universidade Federal Fluminense, Volta Redonda, Brasil. 


\section{INTRODUÇÃO}

O aumento dos níveis de competitividade no mercado tem como uma de suas consequências a busca incessante por redução de custos nas empresas, sejam elas de grande, médio ou pequeno porte. Este fato leva a uma reavaliação de vários conceitos e práticas antes tidas como verdades absolutas. Neste sentido, novas práticas que tem como objetivo reduzir o custo de manutenção são extremamente validas e mudanças de paradigmas são possíveis.

Por meio de vivencia no meio de manutenção e pesquisa de campo em organizações foi percebido que geralmente as grandes empresas dispõem de softwares que reúnem informações e geram ordens de serviço, pedidos de realização de manutenção, para as manutenções corretivas, preventivas, preditivas e também provenientes de inspeção. Entretanto a maioria delas não possui uma metodologia eficaz de planejamento e gestão dos serviços de manutenção, o que leva a falhas. Essas falhas podem ser: o esquecimento de inserir alguma tarefa no cronograma; a falta de priorização de tarefas que leva a faltar tempo para realização de trabalhos mais importantes; a má alocação de recursos; a falta de materiais na hora da manutenção; atrasos provenientes de riscos não geridos; entre outros.

Em alguns casos acontece de dentro de uma mesma empresa planejadores fazem o planejamento da manutenção de maneira diferente, e desta forma setores alcançarem performances diferentes.

Em empresas pequenas a situação é mais complicada, pois além de todos os problemas que as grandes possuem, na maioria dos casos elas não dispõem de recurso para comprar por softwares renomados de gestão de manutenção e acabam fazendo o serviço de maneira manual ou em planilhas pouco organizadas e sem metodologia alguma.

\subsection{Objetivos}

Desenvolver uma metodologia de planejamento e gestão para redução de falhas em projetos de manutenção.

\subsection{Revisão da literatura}

\subsubsection{O que é projeto?}

Para se entender qualquer metodologia de projeto, se faz necessário o entendimento com clareza de o que é um projeto. A seguir são apresentadas algumas definições de gerações diferentes sobre o tema.

Um projeto é uma organização de pessoas dedicadas que visam atingir um propósito e objetivo específicos. Projetos geralmente envolvem gastos, ações ou empreendimentos únicos de alto risco e devem ser completados numa certa data por um montante de dinheiro, dentro de alguma expectativa de desempenho. No mínimo, todos projetos necessitam ter seus objetivos bem definidos e recursos suficientes para poderem desenvolver as tarefas requeridas [1].

TUMAN [1] em 1989 definiu projeto como um empreendimento único, e em sua definição ele ressalta a expectativa de desempenho e a necessidade de objetivos bem definidos, embora seja um conceito de 1989 continua bem atual. Seguindo na linha do tempo, em 2002 VARGAS [2] afirmou que: 
Projeto é um empreendimento não repetitivo, caracterizado por uma sequência clara e lógica de eventos, com início, meio e fim, que se destina a atingir um objetivo claro e definido, sendo conduzido por pessoas dentro de parâmetros predefinidos de tempo, custo, recursos envolvidos e qualidade.

Mesmo depois de cerca de10 anos VARGAS [2] utiliza conceitos parecidos com TUMAN [1], entretanto ele traz um novo conceito de sequência clara e lógica de eventos, esse conceito faz mais sentido no que se diz respeito a planejamento de projetos. Já em 2013 o PMI [3] sintetiza esse conceito e cria uma definição mais simples e resumida.

Projeto é um esforço temporário empreendido para criar um produto, serviço ou resultado exclusivo [3].

Embora o conceito tenha evoluído ao longo do tempo, a essência continua, um projeto é uma determinada tarefa com data de início e fim, com certo recurso disponível e com tarefas que se desencadeiam de certa forma.

\subsubsection{Metodologia de caráter geral}

A metodologia de projeto proposta por Rozenfeld et al. [4] é dividida em macrofases, subdividida em fases e atividades. A Figura 1 mostra as três macrofases: Pré-Desenvolvimento, Desenvolvimento e Pós-Desenvolvimento.

As macrofases de Pré-Desenvolvimento e Pós-Desenvolvimento são fases mais gerais e podem ser aplicadas a diversos tipos de produto com pequenas alterações. Já a fase de Desenvolvimento enfatiza os aspectos tecnológicos que correspondem na definição do que é o produto e na forma de produção, assim tais atividades dependem da tecnologia envolvida no produto [4].

\begin{tabular}{|l|l|l|l|l}
\hline Pré-Desenvolvimento & Desenvolvimento & Pós-Desenvolvimento
\end{tabular}

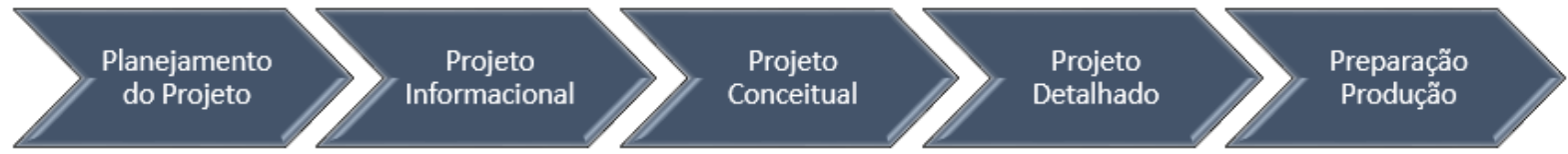

Figura 1- Fases da metodologia de caráter geral.

Fonte: Adaptado pelo autor a partir de ROZENFELD et al. (2006).

O que determina uma fase é a entrega, ou seja, o conjunto de resultados que determinam o patamar de evolução do projeto em desenvolvimento, esses resultados criados em cada fase irão permanecer disponíveis mesmo no momento que a fase é finalizada.

A avaliação dos resultados de cada fase pode ser considerada como um marco de reflexão sobre o andamento do projeto, desta forma se consegue antecipar problemas e guardar lições aprendidas para projetos futuros. Esta avaliação deve ser realizada por meio de um processo formalizado conhecido como transição de fase ou gate [4].

Como descrito no segundo parágrafo desta seção, as atividades e fases dependem no nível de tecnologia e inovação do produto. Como o objetivo deste trabalho é reduzir falhas em um projeto de manutenção, o desenvolvimento da fase de planejamento de projetos será trabalho em detalhes nas próximas seções. 


\subsubsection{Planejamento do projeto}

As atividades do planejamento do projeto, de forma geral, devem empreender esforços no sentido de identificar todas as atividades, recursos e a melhor forma de integra-los para que o projeto siga em frente com o mínimo de erros ou falhas.

O resultado final do planejamento de projeto é o plano de projeto do produto, uma vez que se mostra viável irá guiar a próxima macro fase da metodologia de projeto. É fundamental o bom trabalho nas etapas de pré-desenvolvimento, ou seja, de planejamento sistemático dos projetos pois um bom plano irá permitir uma boa previsão e análise do escopo e riscos, prevenindo falhas durante a execução do projeto [4].

As atividades realizadas nesta fase e a relação entre elas são representadas na Figura 2 e detalhadas nos próximos subitens desta seção.

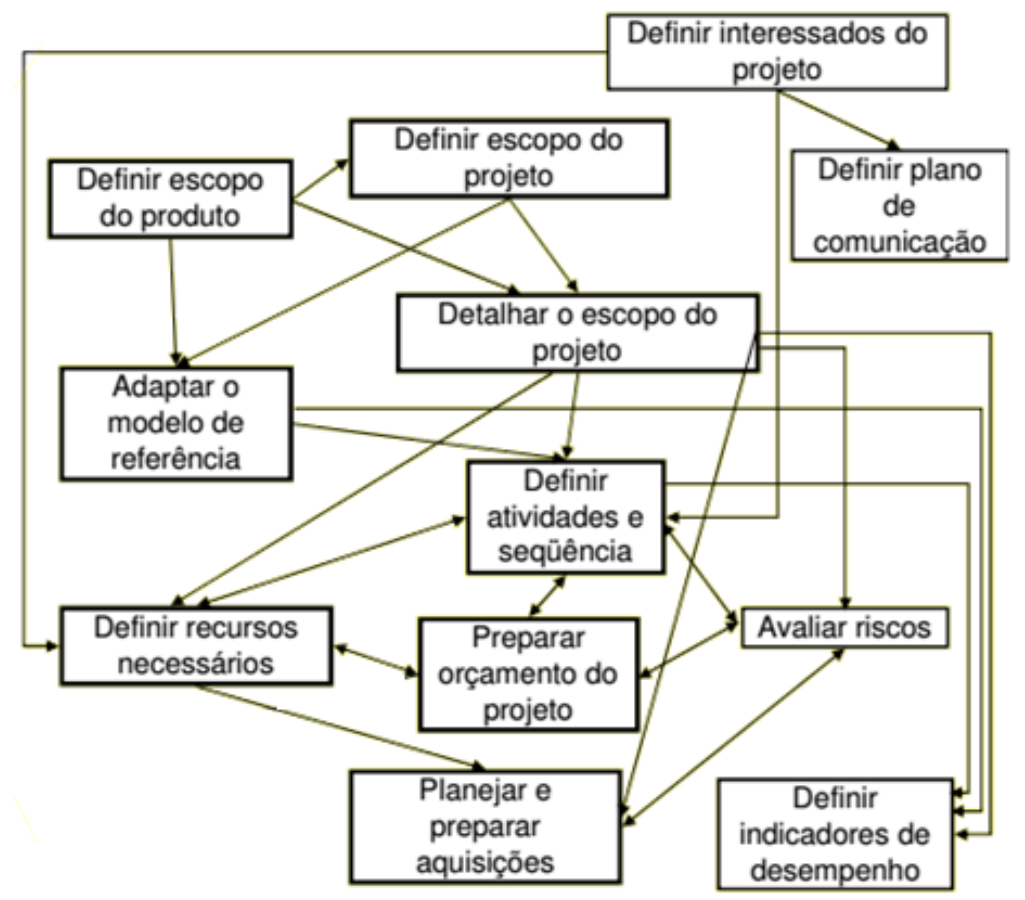

Figura 2- Informações principais e dependência entre as atividades da fase de planejamento do projeto.

Fonte: Adaptado pelo autor a partir do PMI (2013).

\subsubsection{Manutenção preventiva}

Manutenção preventiva é a atuação realizada de forma a reduzir ou evitar a falha ou queda no desempenho, obedecendo a um plano previamente elaborado, baseado em intervalos definidos de tempo [5].

Manutenção preventiva está relacionada com a procura incessante em evitar falhas. Em alguns setores, quando existe risco de segurança, a manutenção preventiva é imperativa pois se deve evitar ao máximo o acontecimento de qualquer falha. A manutenção preventiva tem como função elevar o nível de confiabilidade do equipamento ao mesmo nível de um equipamento novo ou ao mais próximo possível, 
dependendo do caso, a Figura 3 mostra a relação da manutenção preventiva com o desempenho de um equipamento.

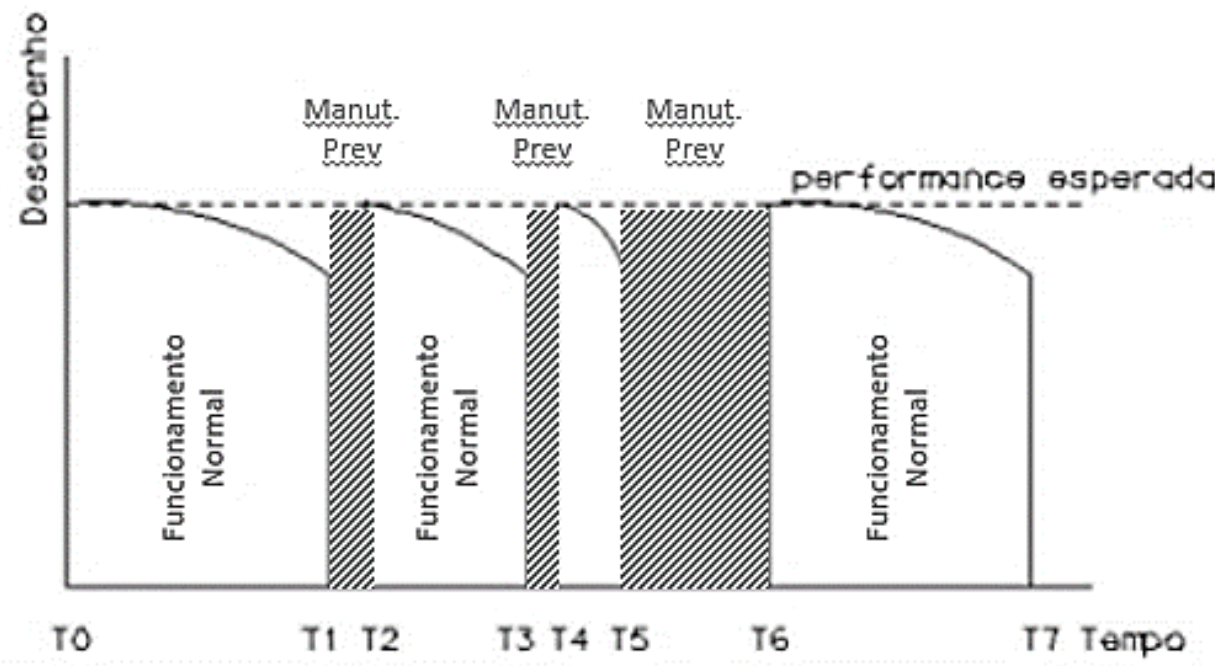

Figura 3- Desempenho e manutenção preventiva.

Fonte: KARDEC (2010).

\section{MATERIAIS E MÉTODOS}

\subsection{Modelo Proposto}

A metodologia proposta nesta seção tem como base a teoria de ROZENFELD et al.[4], acrescidos de pontos de vivencia na área de manutenção. Sendo assim, foi desenvolvida uma metodologia para padronizar o trabalho do planejamento de manutenção utilizando teoria de projeto. A estrutura proposta para metodologia é apresentada na Figura 4. Vale ressaltar que o loop entre as fases de definir cronograma e executar análise de risco deve ser realizado até que seja encontrado o melhor entrelaçamento possível entre as tarefas do projeto, assim quando isto for atingido é hora de seguir para a fase de gerar orçamento.

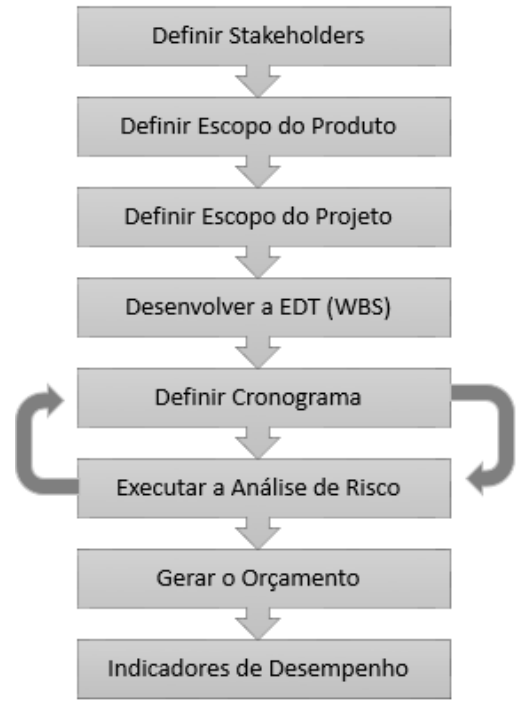

Figura 4- Modelo da metodologia proposta.

Fonte: Autoria própria. 


\subsection{Modelo Proposto}

\subsubsection{Definição de Stakeholders}

Nesta fase se deve lidar com os agentes ou atores que podem manifestar ou sofrer influências sobre o projeto, tanto ao longo de seu planejamento, como em sua realização e após a sua conclusão. Segundo a metodologia proposta, esta atividade é composta de duas etapas: identificar e classificar os stakeholders do projeto

\subsubsection{Identificar os Stakeholders do projeto}

Assim como em outros tipos de projeto, a atividade de manutenção também possui diversos interessados. A Figura 5 mostra com caráter geral quem são os interessados em uma atividade de manutenção.

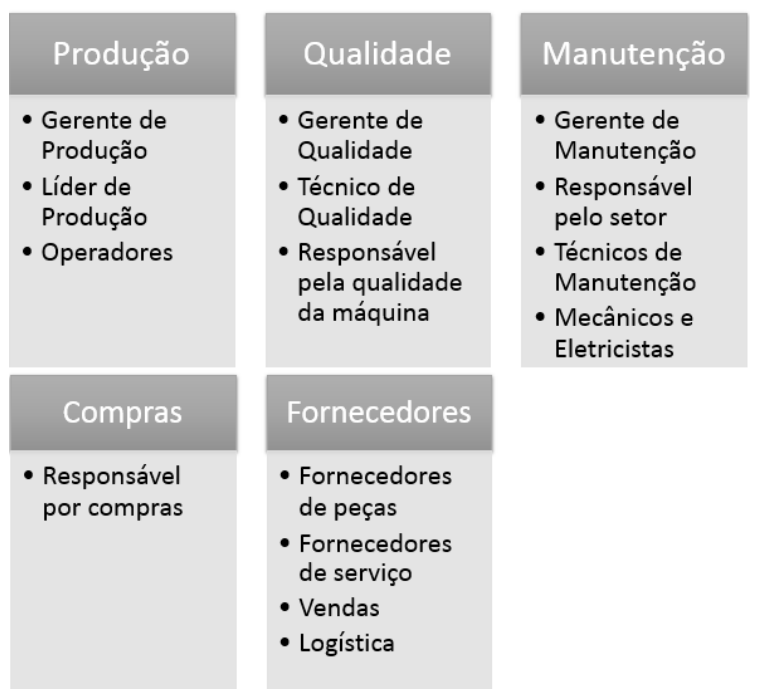

Figura 5- Interessados na atividade de manutenção.

Fonte: Autoria própria.

Dependendo do projeto de manutenção são envolvidos mais ou menos stakeholders, cabe ao gerente do projeto, que na maioria dos casos será o planejador de manutenção, a identificação correta de todos os envolvidos. Vale ressaltar que as partes envolvidas, e sua intensidade de envolvimento podem se alterar ao longo do projeto.

\subsubsection{Classificar os Stakeholders do projeto}

A classificação dos stakeholders deve ocorrer de duas formas, primeiramente se define e classifica a equipe do projeto e em seguida se realiza a classificação das outras partes interessadas.

\subsection{Definição da equipe do projeto}

Geralmente o time de manutenção preventiva será a maior parte da equipe do projeto, mas nesse também podem ser enquadrados mão de obra da qualidade, produção, da própria manutenção, mas vindos de outro setor, e até mesmo mão de obra terceirizada contratada para realização de uma determinada tarefa. 
Assim o gerente do projeto, deve ter o controle de toda mão de obra disponível para o projeto, para que nas fases seguintes ele possa aloca-los da melhor maneira possível.

\subsection{Classificação de outras partes interessadas}

Para a classificação das outras partes interessadas, é proposto um modelo desenvolvido pelo PMI [3]. A Figura 6 mostra o método proposto.

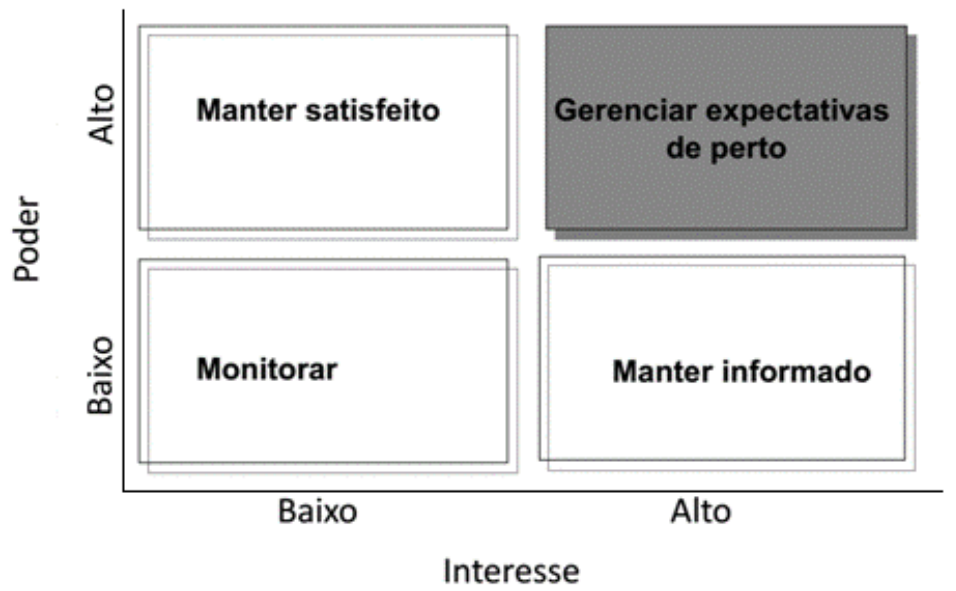

Figura 6- Método de classificação dos stakeholders. Fonte: Adaptado pelo autor a partir do PMI (2013).

Desta forma é possível realizar a classificação de todos os stakeholders, e assim entender quais são críticos para o projeto, sabendo precisamente quais ações devemos tomar com cada um deles para que o projeto seja um sucesso e satisfaça a todos.

\subsubsection{Definir Escopo do Produto}

Nesta fase, a importância é entender o que se espera do projeto e levantar as delimitações do produto que serão importantes para as fases seguintes. Essa etapa na metodologia proposta consiste basicamente em entender três pontos:

- Delimitação de data: A delimitação de data se torna importante para conciliar os recursos materiais e humanos necessários no dia correto da manutenção.

- Delimitação de tempo: A delimitação de tempo é fundamental, pois ela que irá determinar todo o cronograma da manutenção, é chamado também de janela de manutenção preventiva.

- Resultados esperados: Como foi realizado a classificação das partes interessadas no tópico anterior, já se sabe quem são os principais stakeholders. É de fundamental importância entender o que os eles esperam do projeto, para que tudo possa ser acertado antes do início das atividades, e assim ao final sejam satisfeitos os interesses principais do projeto. 


\subsubsection{Definir Escopo do Projeto}

A fase de definir o Escopo do projeto é fundamental para o sucesso do projeto, uma vez que nela é descrita "como" o produto será obtido, premissas assumidas, entre outras. Portanto a metodologia para esta fase, mostrada na Figura 7, deve ser aplicada com o máximo de detalhes possíveis, uma vez que as próximas fases estão diretamente ligadas a ela.

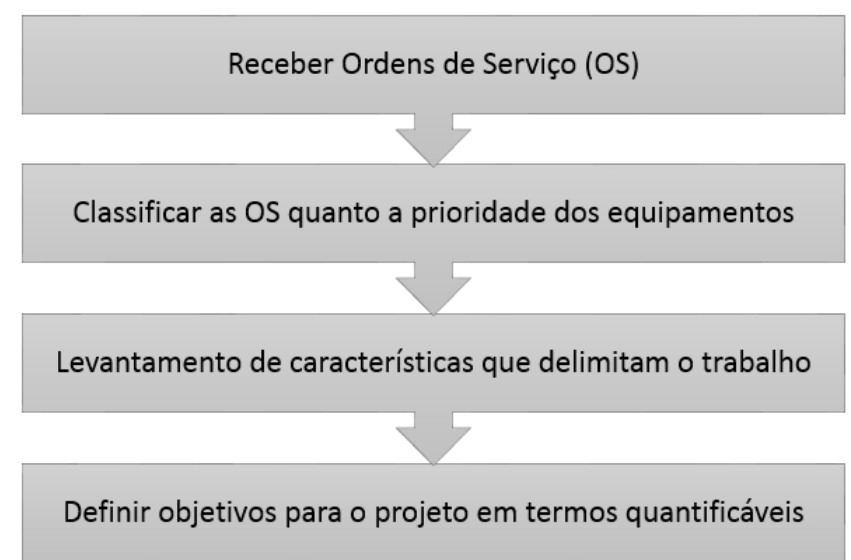

Figura 7- Metodologia para a fase de definir Escopo do Projeto.

Fonte: Autoria própria.

\subsubsection{Desenvolver a Estrutura de Decomposição do Trabalho (EDT)}

O objetivo desta fase consiste em agrupar as Ordens de Trabalho, recebidas e classificadas, por deliverable.

A metodologia proposta coloca a manutenção do equipamento como Produto, os entregáveis (deliverables) são as manutenções nos conjuntos da máquina, já os pacotes de trabalho são as manutenções nos subconjuntos dos equipamentos. Sendo assim a manutenção será dividida de uma forma lógica e com possibilidade de verificação contínua. A Figura 8 mostra essa divisão em um exemplo de EDT em manutenção.

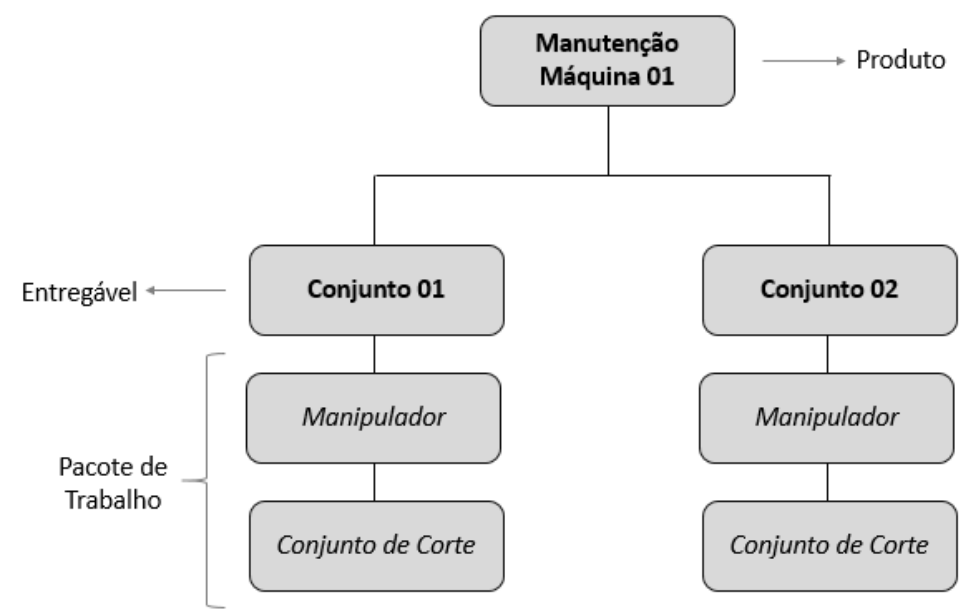

Figura 8- Exemplo de EDT de manutenção

Fonte: Autoria própria. 
Vale salientar que dentro de cada pacote de trabalho devem existem várias tarefas para serem executadas. Desta forma, é aconselhado ao planejador possuir um modelo de referência padronizado para cada pacote de trabalho com as tarefas e também outras informações importantes na hora de realizar o cronograma, estudo de risco, estudo de custo e também para enviar o serviço ao executante. As informações fundamentais que devem estar contidas no modelo de referência para a realização de um planejamento de qualidade são listadas a seguir:

- Descrição;

- $\quad$ Carga de Trabalho do Pacote de Trabalho (hrs);

- $\quad$ Número de mão de obra necessária;

- Tipo de mão de obra necessária;

- Duração do Pacote de Trabalho;

- Descrição das Tarefas do Pacote de Trabalho;

- Materiais necessários para realizar as tarefas;

- Valor total dos materiais.

\subsubsection{Definir Cronograma}

Para realizar a definição do cronograma de uma forma analítica e não simplesmente por palpites e convicções, é necessária a utilização do gráfico de Gantt, utilizado por muitas empresas para realizar o planejamento de ações de manutenção.

Entretanto a metodologia do autor, propõe a utilização do gráfico de Gantt em conjunto com o Método do Caminho Crítico (CPM). A forma de aplicação da metodologia para esta fase segue a seguinte rotina:

1. Utilizar a EDT como base para o cronograma;

2. Definir as datas de início e fim para cada pacote de trabalho.

3. Selecionar o recurso os recursos humanos para cada pacote de trabalho.

4. Aplicar os vínculos e limitações entre os pacotes de trabalhos.

5. Aplicar o método CPM no projeto;

6. Analisar o caminho crítico do projeto;

7. Aplicar e analisar o gráfico de Gantt;

8. Buscar maneiras de amenizar o caminho crítico com a visualização do gráfico de Gantt;

9. Repetir o ciclo até encontrar a melhor estrutura possível para o cronograma.

\subsubsection{Executar a análise de risco}

A fase da análise de risco é considerada um diferencial da metodologia, visto que a maioria das empresas não a aplica ou aplica sem uma metodologia eficiente. $O$ correto levantamento de informações e, posteriormente análise e gestão do plano de ação irão diminuir a probabilidade do aparecimento de surpresas, agradáveis ou desagradáveis, durante a execução do projeto. A Figura 9 mostra como a metodologia proposta pelo autor deste trabalho deve ser trabalhada. 


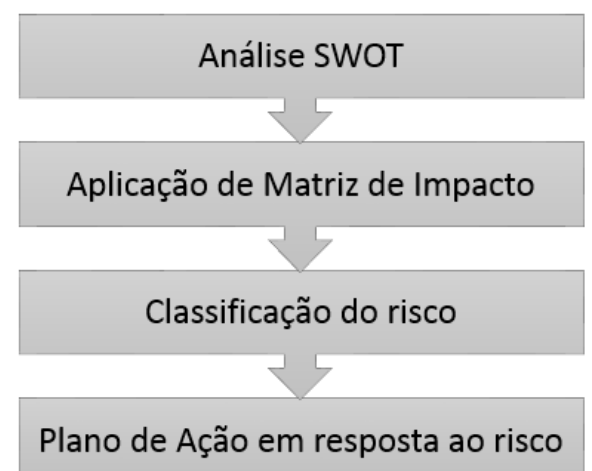

Figura 9- Etapas da fase de análise de risco.

Fonte: Autoria própria.

\subsubsection{Gerar o Orçamento}

Como a metodologia é focada em manutenção, foi escolhido o método Bottonup que também é chamado de estimativa de baixo para cima, nela se tem como ponto de partida os custos de cada atividade do projeto e se agrupa progressivamente esses resultados pontuais até chegar à estimativa de custos de recurso para todo projeto.

Este método está bem alinhado com o que a metodologia propõe, uma vez que no modelo de referência de pacote de trabalho possuirá a classificação da mão de obra, o número de recursos humanos, a duração de cada atividade, os materiais e respectivos custos. Desta forma o método Botton-up se torna perfeito e de fácil aplicação para esta metodologia, basta apenas o planejador utilizar os dados já disponíveis para fazer a análise de custo e calcular quanto o projeto de manutenção irá gerar de custos para empresa. Como cada custo estará detalhado individualmente, também será facilitada a tarefa de excluir alguma tarefa caso seja necessário a redução de custos no projeto.

\subsubsection{Indicadores de Desempenho}

Para gestão e controle do projeto, é fundamental a utilização de indicadores de desempenho para garantir que o planejamento da manutenção está de acordo com a realidade. O planejamento ideal não deve ser nem muito mais e nem muito menos do que o tempo necessário para realizar as tarefas na realidade, quanto mais maduro é o planejamento mais justo e condizente com a realidade ele é. Para situação de cada empresa podem ser propostos indicadores diferentes, o importante é que dentro de uma mesma empresa os indicadores sejam o mesmo, para efeito de comparação.

\section{RESULTADOS E DISCUSSÃO}

A definição de stakeholders é algo que acontece de maneira informal nas empresas, assim nem sempre todos stakeholders são identificados e geridos da forma correta. Desta forma, com a aplicação da metodologia se espera um gerenciamento de qualidade dos stakeholders, entendendo quais são os críticos e o que eles esperam do projeto de manutenção. Para que uma vez alinhados, os grandes objetivos sejam realizados e o planejamento facilitado.

Em relação ao Escopo do Produto e Escopo do Projeto, geralmente as empresas os aplicam, entretanto sem padronização e sequencia previa, são informações que o planejador de manutenção levanta ao longo do planejamento. 
Entretanto devido à falta de padrão muitas vezes são esquecidas algumas informações que podem vir a ser fundamentais para a realização do projeto, e o mesmo pode sofrer severos atrasos ou remanejamentos. Isto posto, a proposta da metodologia de sequenciamento do trabalho será de grande importância para a padronização do projeto de manutenção, evitando assim falhas de planejamento que são tão comuns no dia a dia das empresas.

$\mathrm{Na}$ fase de desenvolvimento da EDT, o grande diferencial proposto pela metodologia é o modelo de referência padronizado para cada pacote de trabalho com as tarefas, desta forma o planejador irá possuir um banco de dados com todos os pacotes de trabalho possíveis para seu setor e na hora do planejamento da manutenção ele possuirá todas as informações necessárias para realizar o planejamento de forma ágil e precisa, contendo inclusive informações de custo. Outra vantagem esperada é que o trabalho do executante da tarefa seja facilitado, uma vez que a ordem de serviço já será direcionada para ele com todas as tarefas especificadas e sequenciadas da melhor forma possível, fato este que não ocorre na maioria das empresas, onde é necessária a experiência do mantenedor para a realização do serviço na sequência correta.

Quando se trata de definição de cronograma, a metodologia trabalha com duas ferramentas conhecidas no mercado, o gráfico de Gantt e o Método do Caminho Crítico (CPM). Entretanto se espera conseguir o desenvolvimento e aplicação de tais ferramentas de maneira interativa, ou seja, que não seja necessário o cálculo manual, isto se torna válido pois dependendo do tamanho do projeto, aplicar o método CPM se torna uma tarefa complicada. Tal aplicação também é válida pois não são todas as empresas que possuem a licença de um software de gestão de projetos para auxiliar na manutenção, na região Sul do estado do Rio de Janeiro, onde este trabalho foi desenvolvido, isto é muito raro.

$\mathrm{Na}$ fase de análise de risco do projeto a metodologia propõe algo que na maioria das vezes não é realizado em projetos de manutenção, se espera que a análise de risco combinando SWOT, Matriz de Impacto e Plano de Ação apesente grande influência sobre o sucesso do projeto, aumentando consideravelmente a previsibilidade de possíveis impactos.

Com a utilização da técnica Bottom up para previsão do orçamento do projeto se tem pretensão de convergência entre custo planejado e custo realizado, tal técnica é minimalista e a sua aplicação será facilitada pelo software desenvolvido, assim o orçamento será calculado de forma automática e o trabalho do planejador será muito auxiliado.

Os indicadores de desempenho têm como objetivo mostrar o quão assertivo o projeto de manutenção foi, o planejador possuirá um banco de dados com o desempenho de todas as manutenções, facilitando assim a comparação entre projetos e possibilidade de utilização de melhores práticas dos projetos de maior rendimento, desta forma os indicadores poderão contribuir com o desenvolvimento e a qualidade do planejamento de projetos na empresa. 


\section{CONCLUSÃO}

A proposta de contribuir de forma significativa para a tarefa de planejamento de manutenção através da modificação e adaptação de modelos existentes na literatura foi alcançada de maneira satisfatória. Uma vez que existe uma real contribuição para a literatura, com a criação de um novo e diferente método de planejamento de manutenção, que auxilia o planejador a organizar as tarefas de uma maneira clara, simples, de fácil aplicação e com conceitos firmes que permitem a análise e gestão de riscos e definição de cronogramas em projetos de forma robusta.

Esta metodologia converge, em relação ao sequenciamento e organização, com a de ROZENFELD et al [4], entretanto a forma de aplicação é realizada de maneira diferente, com foco total em manutenção. A aplicação da ferramenta SWOT, seguido da matriz de impacto na análise de risco de uma manutenção, é um diferencial nesta metodologia.

Portanto conclui-se que a metodologia atende os requisitos de ser de fácil aplicação e sequenciada, logo está pronta para a próxima fase, ser aplicada em um programa computacional e inserida em ambiente industrial para efetivamente ser testada.

\section{Agradecimentos}

À CAPES pelo apoio financeiro recebido no ano de 2017.

\section{REFERÊNCIAS}

1 TUMAN, G. J. Development and implementation of effective project management information and control systems. New York: Van Nostrand Reinhold; 1983.

2 VARGAS, R.V. Análise do valor agregado em projetos: revolucionando o gerenciamento de custos e prazos. Rio de Janeiro: Brasport; 2002.

3 PROJECT MANAGEMENT INSTITUTE. Um guia do conjunto de conhecimentos em Gerenciamento de Projetos (Guia PMBoK). 5.ed. Pennsylvania: Four Campus Boulevard: PMI; 2013.

4 ROZENFELD, H., et al. Gestão de Desenvolvimento de Produtos Uma referência para a melhoria do processo. Rio de Janeiro: Saraiva; 2006.

5 KARDEC, A e NASCIF, J. Manutenção: função estratégica. 3. ed. Rio de Janeiro: Qualitymark; 2010. 